\title{
Pace of movement: the role of single neurons in the subthalamic nucleus
}

\author{
Ariel Tankus, PhD,,-4 Anat Mirelman, PhD, ${ }^{2-4}$ Nir Giladi, MD, ${ }^{3-5}$ Itzhak Fried, MD, PhD, ${ }^{1,3,6}$ and \\ Jeffrey M. Hausdorff, PhD $2,4,7-9$ \\ ${ }^{1}$ Functional Neurosurgery Unit, ${ }^{2}$ Center for Study of Movement, Cognition, and Mobility, ${ }^{5}$ Department of Neurology, Tel Aviv \\ Sourasky Medical Center; ${ }^{3}$ Department of Neurology and Neurosurgery, Sackler Faculty of Medicine, and ${ }^{4}$ Sagol School of \\ Neuroscience, Tel Aviv University, Tel Aviv, Israel; ${ }^{6}$ Department of Neurosurgery, University of California, Los Angeles, California; \\ ${ }^{7}$ Department of Physical Therapy, Sackler Faculty of Medicine, Tel Aviv University, Tel Aviv, Israel; and 'Rush Alzheimer's \\ Disease Center and ${ }^{9}$ Department of Orthopedic Surgery, Rush University Medical Center, Chicago, Illinois
}

OBJECTIVE The ability to modulate the pace of movement is a critical factor in the smooth operation of the motor system. The authors recently described distinct and overlapping representations of movement kinematics in the subthalamic nucleus (STN), but it is still unclear how movement pace is modulated according to the demands of the task at the neuronal level in this area. The goal of this study was to clarify how different movement paces are being controlled by neurons in the STN.

METHODS The authors performed direct recording of the electrical activity of single neurons in the STN of neurosurgical patients with Parkinson's disease undergoing implantation of a deep brain stimulator under local anesthesia while the patients performed repetitive foot and hand movements intraoperatively at multiple paces.

RESULTS A change was observed in the neuronal population controlling the movement for each pace. The mechanism for switching between these controlling populations differs for hand and foot movements.

CONCLUSIONS These findings suggest that disparate schemes are utilized in the STN for neuronal recruitment for motor control of the upper and lower extremities. The results indicate a distributed model of motor control within the STN, where the active neuronal population changes when modifying the task condition and pace.

https://thejns.org/doi/abs/10.3171/2018.1.JNS171859

KEYWORDS movement pace; single-unit recording; neuronal recruitment; hands and feet motor control; subthalamic nucleus; Parkinson's disease; functional neurosurgery

$\mathrm{H}$ UMANS utilize their lower and upper extremities in completely different manners. Although the human motor control networks for the feet and hands share multiple brain areas, such as the primary and supplementary motor areas, premotor cortex, and subthalamic nucleus (STN), ${ }^{29}$ physiological network differences have been described at the subregion level, such as the somatotopic organization of the STN, $, 17,20,25$ with active movements of the wrist, arm, shoulder, leg, and foot, where the arm is represented more laterally than the leg. Our study examines differences between lower- and upper-extremity representations in the STN.

In the intact monkey, the activity of over half the cells examined in the STN was modulated in response to passive movements of individual contralateral body parts, ${ }^{30}$ mostly to passive joint rotation produced by muscle palpation, but in some cases also in response to light touch. Within the rostrodorsal zone, a lateral region was found to contain neurons that responded to arm movements; a more medial region, to leg movement; and more dorsally and rostrally, to orofacial movements. In humans with Parkinson's disease (PD), a motor area related to passive movements was found in the dorsolateral part of the STN, with leg-related cells located medially and centrally and armrelated cells found more diffusely. ${ }^{27}$ Responses to active and passive movements (e.g., clenching the fist, pointing, foot tapping, horizontal and vertical saccades, jaw movement, and tongue protrusion) were also demonstrated in the STN of patients with PD. ${ }^{10}$

Subthalamic neuronal activity has been related to mul- 
tiple motor parameters of both upper and lower extremities. Upper-extremity motor execution, motor inhibition, and error monitoring have been found to activate 3 subpopulations of subthalamic neurons, ${ }^{2,3}$ as has Parkinsonian tremor. ${ }^{1,20}$ Upper-extremity parameters include grip force, ${ }^{18}$ movement onset, and movement direction. ${ }^{7}$ Participation of the STN in lower-extremity control was shown by stimulation studies in which stimulation improved gait and postural symptoms of PD, sometimes to an almost normal level ${ }^{4,11,13}$ (see Piper et al..$^{19}$ for a review). STN stimulation, combined with levodopa, improved gait speed, reduced gait variability (enhanced stability), and yielded lower (better) Unified Parkinson's Disease Rating Scale (UPDRS) scores. ${ }^{8}$ We have recently shown that the STN encodes the kinematic parameters of movement-orientation, angular velocity, and acceleration-and is involved in motor coordination. ${ }^{25}$ These findings demonstrate that the STN is a key region in the regulation of both upper- and lower-extremity movement control.

Despite the aforementioned literature, the question of how subthalamic neurons are recruited for motor control of the hand and feet is still unclear. In particular, it is not yet clear whether different paces of movement involve the same population of controlling neurons or require recruitment of additional units or whether firing modulation by itself can explain the change in movement pace. We hypothesize that a drastic change in movement pace is usually not a reflection of mere performance of the same movement slower or faster, but commonly reflects a change to the "nature" of movement, for example, from gross to fine motor skills. We therefore expect different neuronal populations to control the different paces. Here, we extend our previous study ${ }^{25}$ by exploring this issue at the single cell level of the STN.

As deep brain stimulation (DBS) is a surgical treatment for the motor signs of PD, we believe that characterizing the physiology of the various motor-related neuronal populations is a first step that will, in the future, inform better fitting of the treatment to the signs of the disease of the individual patient and avoid side effects to neighboring populations in more focused DBS procedures.

\section{Methods}

\section{Patients and Electrophysiology}

Participants in this study were 10 patients with PD who were undergoing implantation of DBS electrodes for clinical reasons. The patients' mean age was 59.2 years (SD 11.7 years), and their mean duration of PD was 11.1 years (SD 4.2 years). All were right-handed, and 8 were male. The clinical procedure and microelectrode recordings were previously described ${ }^{25}$ and so was spike sorting. ${ }^{26}$ All studies were approved by the institutional review board of Tel Aviv Medical Center and conformed to its guidelines. All patients provided written informed consent.

\section{Experimental Paradigms}

Intraoperatively, patients performed unipedal and bipedal (alternating) tapping against a surface, and similarly, unimanual and bimanual (alternating) tapping, also against a surface. Although tapping, even if alternating, does not necessarily reflect gait, the intraoperative supine position constrains the task, and this setup was used as a rough approximation.

Each type of tapping was repeated at 3 internally generated paces: patient self-selected ("normal"), slow, and fast paces. Patients were first instructed to perform the tapping task. By intention, the instructions did not refer to any pace. The patients selected the pace by themselves, and none of them asked about it. Next, patients were instructed to perform the task faster than before. Finally, they were asked to perform the task slower than in the first part. During the tasks, patients wore small, light, wireless measurement devices on both wrists and the dorsal surfaces of both feet (Opal monitors; APDM Inc.). These devices recorded kinematics (acceleration, angular velocity, and orientation) in synchrony with the neuronal recording.

\section{Data Analysis}

The modeling of the firing rate function as a linear combination of each triaxial kinematic parameter-orientation, angular velocity, and acceleration (cross-validated linear regression) - and the evaluation of the nonlinear relations between firing and kinematics based on entropy correlation coefficients ${ }^{28}$ were described in our previous study. ${ }^{25}$ The entropy correlation coefficient is independent of the length of the time series, thus "normalizing" the longer duration of slower movements. Hence, the entropy correlation coefficient of long time series can be directly compared with those obtained from shorter series. Because the distribution of entropy correlation coefficients is not normal, the Mack-Skillings test ${ }^{14}$ served for nonparametric 2-way unbalanced analysis of variance (ANOVA).

For large samples, the sampling distribution of the median is approximately normal. Therefore, when comparing the median entropy correlation coefficients between 2 conditions (e.g., normal-pace movements and slow movements), we employed the parametric paired-sample t-test. The same statistical test served to compare the average percentage of task-related units (over all patients) between conditions.

\section{Results}

We recorded the activity of 89 single units in the STN of 10 PD patients who were undergoing implantation of a deep brain stimulator for treatment of their motor symptoms. Figure 1 compares the average cycle times of the repetitive movements at the different paces. The figure demonstrates that the cycle time of the repetitive movements was indeed significantly different during the slow, normal, and fast tapping paces (Fig. 1A and B). This was the case independent of the limb or limbs participating in the movement. Moreover, it appears that for each tapping pace, patients selected similar cycle times for tapping with the hands as with their feet (Fig. 1C).

In our earlier work,,$^{25}$ we found that in $43 \%$ (38/89) of the recorded units, the correlation between the firing rate and its kinematics-based linear estimator was significant, explaining a considerable percentage of the variance $\left(\mathrm{r}^{2}>\right.$ 0.30 ). In addition, we found significant relations between 

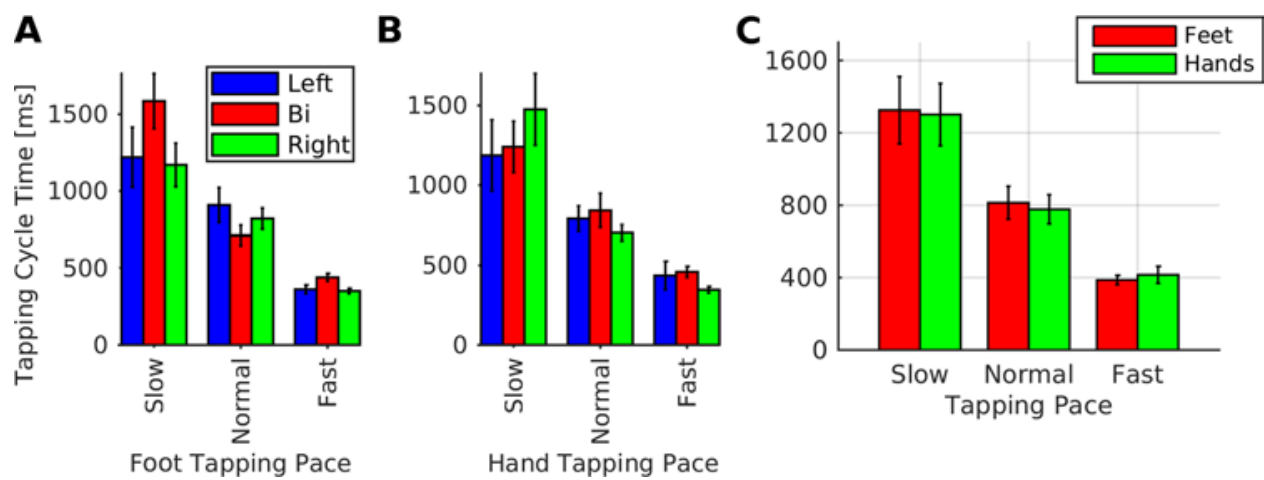

FIG. 1. Limb movement behavior in each experimental condition. The average tapping cycle time was significantly longer in the slow tapping pace than in the normal pace and significantly longer in the normal pace than in the fast condition, independent of the limb(s) involved, as expected (2 paired-sample t-tests, $p<0.05$ in all tests). A: Foot tapping. B: Hand tapping. C: Average cycle time for the slow, normal, and fast tapping paces, for foot (red) and hand (green) movements. The pace of feet and hand movements was similar. $\mathrm{Bi}=$ bipedal or bimanual; $\mathrm{ms}=$ milliseconds. Error bars indicate standard errors (SEs).

kinematics and spiking activity in $93 \%$ (83/89) of recorded STN units based on the entropy correlation coefficient (see Methods).

In the present study, we found that the entropy correlation coefficient was significantly affected by both the tapping pace (i.e., slow, normal, or fast) and the limb (ipsi- vs contralateral) during both foot movements (pace: $\mathrm{p}<0.0001$; limb: $\mathrm{p}<0.0001$; the Mack-Skillings test for nonparametric 2-way unbalanced ANOVA) and hand movements (pace: $\mathrm{p}<0.0001$; limb: $\mathrm{p}<0.0001$; Fig. $2 \mathrm{~A}$ and B). Notice, however, that independent of the limb, the entropy correlation coefficient was larger during normalpace movements than during slow movements (see also Fig. 2C), both for hands ( $p=0.017$; paired-sample t-test) and for feet $(\mathrm{p}=0.043)$. Thus, the recorded neurons apparently encode less information in slow movements than in normal-pace ones.

So far, we examined the change in entropy correlation coefficient for each neuron by itself. Does this mean that for both foot and hand movements the population as a whole encodes less information for slow movements than for normal-pace movements? To address this question, we examined the percentage of units recruited for the tapping task under each condition. This percentage changed as a function of movement pace, as can be seen by the stairlike structure in Fig. 2D and E. Even though this type of structure is common to hands and feet, the order of the "stairs" is different (note the different labeling of the yaxes in Fig. 2D and E). For foot movements, normal-pace movements yielded the maximum percentage of recruited units, slow movements yielded significantly less (normal $>$ slow: $\mathrm{p}=0.035$, paired-sample t-test; see Methods), and fast movements yielded the least (slow $>$ fast: $p=0.0065$ ). In contrast, for hand movements, slow movements were the ones recruiting the maximum percentage of units; normal-pace movements, significantly less (slow > normal: $p$ $=0.0037$ ); and fast movements, the least (normal $>$ fast: $p$ $=0.0064$ ). Thus, for foot movements, the change of pace from normal to slow results in fewer units recruited for the task (Fig. 2F, blue), each unit carrying less information on the slow movement task (Fig. 2C, blue). For hand movements, however, each unit carried less information on the slow movements (Fig. 2C, red), but more units were recruited for the task (Fig. 2F, red).

Direct comparison of the percentage of units recruited to control hand and foot movements in each pace reveals that for the slow $(\mathrm{p}=0.22)$ and fast $(\mathrm{p}=0.51)$ paces there was no significant difference between the upper and lower limbs. However, as Fig. 2F shows, during normal pace, the difference between feet and hands was significant ( $p$ $=0.001$; feet $>$ hands, paired-sample t-test). Similarly, feet and hands did not differ significantly in their entropy correlation coefficient during slow $(\mathrm{p}=0.17)$ and fast $(\mathrm{p}=$ 0.12 ) paces, but they did during normal pace $(p=0.039$; feet $>$ hands, paired-sample t-test).

\section{Discussion}

In this study, we report disparate pace-dependent recruitment schemes for subthalamic neurons controlling hand movements compared with those controlling the feet. The information encoded by the activity of each controlling neuron decreased when moving from a normal, self-selected pace to a slow tapping pace, in both lowerand upper-extremity movements (see Fig. 2A-C). For foot movements, this decrease was accompanied by a reduction in the percentage of recorded neurons recruited for the task (see Fig. 2D-F), leading to an overall reduction in the task-related information represented by the population as a whole. In contrast, for hand movements, the percentage of recruited neurons we recorded increased, in what might be a compensatory mechanism that uses more neurons, each carrying less information. Thus, the population as a whole may avoid significant reduction in the amount of information about slow movements in comparison with normal-pace ones. Slow hand movements may serve, for example, in fine motor skills, and may thus require finer control, that is, increased neuronal activity or a shift in neuronal activity between cell populations. Slow movements also result from deficits shown by the patients, including bradykinesia.

Our finding of different neuronal populations each spe- 


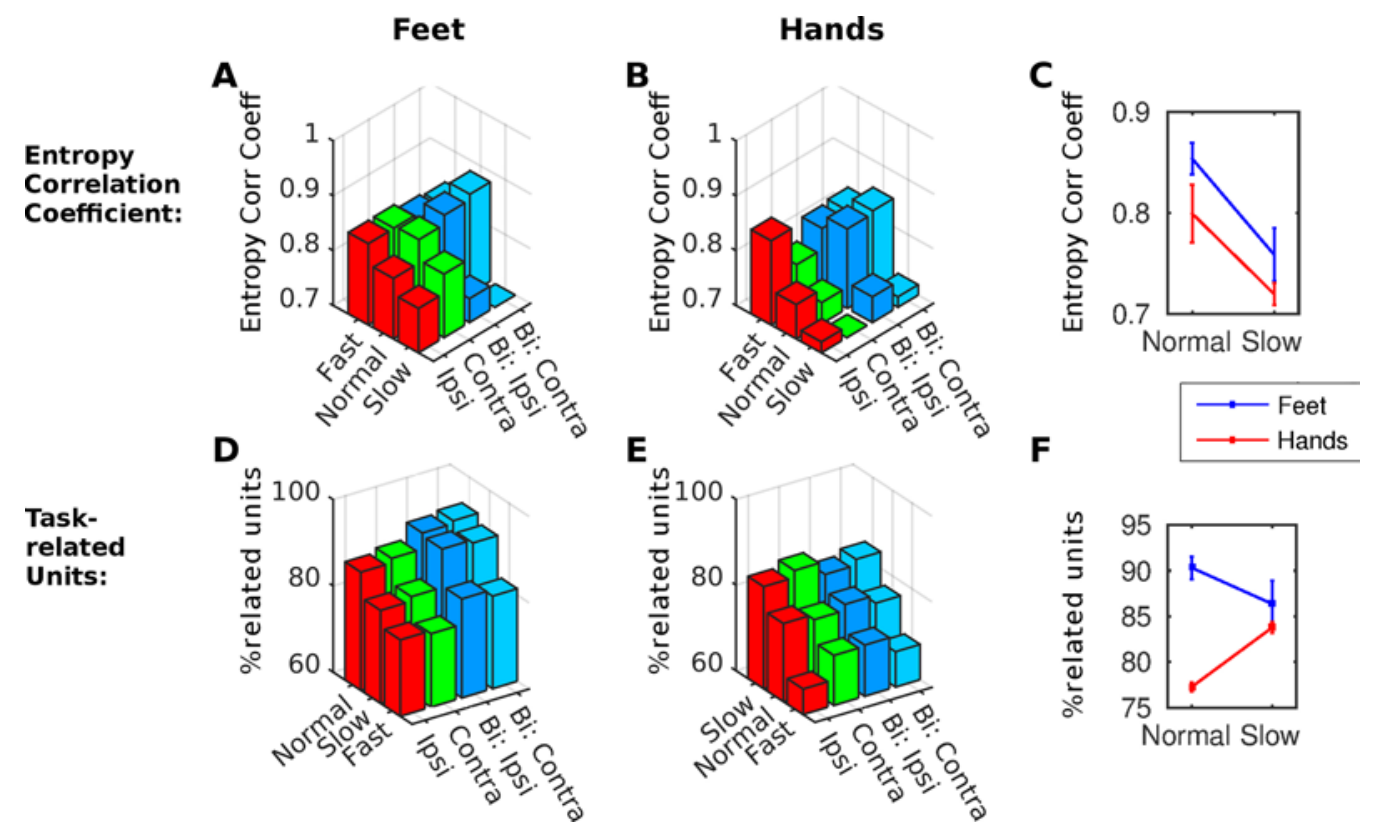

FIG. 2. Median entropy correlation coefficient and average percentage of task-related units, over all patients, for each tapping pace and each limb. A-C: Entropy correlation coefficient. The median entropy correlation coefficient varied significantly with both pace and limb, for both foot $(A)$ and hand $(B)$ movements. Note in particular the reduction in information when moving from normal pace to slow pace, independently of the limb. This transition is summarized in $\mathrm{C}$ : the entropy correlation coefficient decreases for both foot (blue) and hand (red) movements. D-F: Percentage of task-related units. The average percentage of task-related units shows a stair-like structure. For foot tapping (D), more neurons are recruited during normal tapping, fewer during slow tapping, and even fewer during fast tapping. For hand tapping $(E)$, slow tapping recruits the largest amount of units; normal tapping, fewer; and fast pace, the least, independent of the limb. (Note the different labeling of the pace axes in $\mathrm{D}$ and $\mathrm{E}$.) The percentages of recruited neurons in normal and slow movements are summarized in F. Neuronal recruitment schemes differ between feet (blue) and hand (red) movements. For foot movements, the transition decreases the amount of participating neurons. Combined with the decrease in information encoded by each neuron $(C)$, the overall result is reduced encoded information for slow foot movements. In contrast, for hand movements the decrease in encoded information $(C)$ is compensated for by recruiting more neurons for the task (increase in percentage of recruited neurons). $\mathrm{Bi}=$ bipedal or bimanual; coeff $=$ coefficient; contra $=$ contralateral; corr $=$ correlation; ipsi $=$ ipsilateral.

cifically involved in a certain pace of movement extends existing evidence in the mouse spinal cord, where ablation of inhibitory V0 neurons leads to a quadrupedal hopping at all frequencies of locomotion, but with a differential effect at different locomotor frequencies: lack of left-right pattern at low frequencies, mixed coordination at medium frequencies, and left-right alternation at high locomotor frequencies. ${ }^{22}$ Similarly, switches in spinal circuits during increases in locomotor speed were provided by studies of swimming in larval zebrafish. ${ }^{12}$

The aforementioned differences in the neuronal recruitment scheme between foot and hand movements cannot be explained by differences in tapping paces, as the average pace of foot movements was similar to that of hand movements (Fig. 1). The recruitment differences are, however, consistent with existing evidence for the role of the STN in the control of automatic behavior ${ }^{9}$ and task switching, ${ }^{15}$ because automatic behavior most closely reflects movements in the normal, patient-selected pace. As we have shown, for this type of movement the STN encodes more kinematic information than for slow movements, implying greater subthalamic involvement in the control of automatic movements. Moreover, the reported differences also suggest that an external electrical stimulation to the STN will likely yield distinct effects at each movement pace. This may thus indirectly explain, at the single cell level, the differences in step length and velocity induced by DBS in normal-pace versus fast gait. ${ }^{4}$

The difference in percentage of recruited units for hand and foot movements is about $12 \%$ (Fig. 2F, normal pace). This percentage is similar to percentages reported in the literature on human neurophysiology to describe various phenomena-for example, units related to speech in the temporal and frontal lobes ${ }^{24}(8 \%, 49$ units), visuomotor units in the parahippocampal gyrus ${ }^{23}$ (9\%, 11 units), or pure bipedal units in the $\operatorname{STN}^{25}$ (11\%, 10 units). We therefore conclude that disparate pace-dependent recruitment schemes exist for the STN neurons that control hand and foot movements.

Understanding the neuronal recruitment schemes for different types of movements (e.g., different paces) may, in the future, assist neurosurgeons in the optimal targeting of DBS electrodes to achieve multiple goals. First, it may help in targeting areas with neuronal activity related to specific symptoms, for example, slow gait. Second, it may assist in avoiding DBS side effects, such as the aforementioned difference in the influence of stimulation on fast versus normal paces. Third, more focused stimulation may 
be developed that will combine the two benefits: it will target a specific group of neurons to treat a specific symptom while avoiding other neurons that, when stimulated unnecessarily, produce side effects. Fourth, closed-loop $\mathrm{DBS}^{21}$ may be tailored for different types of movement based on the different neuronal populations identified.

Our results suggest that different movement paces may lead to distinct motor control schemes in the STN, as opposed to a single control scheme that takes velocity or pace into account, as is the case, for example, in the primary motor cortex. ${ }^{5,6,16}$ Nevertheless, as we recorded from a random sample of cells, and as the size of the sample is small relative to the whole neuronal population that controls movement within the STN, we must bear in mind that the distinct schemes that we observed may in fact represent different aspects of a more complex unified scheme. Future research with a larger cohort may be necessary for a more complete understanding of the underlying mechanisms.

\section{Conclusions}

Change of pace of movement is achieved by recruitment of the corresponding subthalamic neuronal population. The recruitment schemes for controlling hand movements differ from those for controlling the feet. Our findings are thus an important step on the way to deciphering the neuronal mechanisms involved in the recruitment of subthalamic neurons for the motor control task. In the future, these results may help to facilitate more focused implantation of DBS electrodes in patients with movement disorders, targeting specific symptoms related to movement kinematics.

\section{Acknowledgments}

This work was supported by the I-CORE Program of the Planning and Budgeting Committee and the Israel Science Foundation (grant no. 51/11 to I.F.), a Tel Aviv Medical Center Excellence grant (to A.T.), the W. Schreiber fund (to A.T.), and the National Institute for Psychobiology in Israel-founded by the Charles E. Smith family (to A.T.).

\section{References}

1. Amtage F, Henschel K, Schelter B, Vesper J, Timmer J, Lücking $\mathrm{CH}$, et al: Tremor-correlated neuronal activity in the subthalamic nucleus of Parkinsonian patients. Neurosci Lett 442:195-199, 2008

2. Bastin J, Polosan M, Benis D, Goetz L, Bhattacharjee M, Piallat B, et al: Inhibitory control and error monitoring by human subthalamic neurons. Transl Psychiatry 4:e439, 2014

3. Benis D, David O, Piallat B, Kibleur A, Goetz L, Bhattacharjee $\mathrm{M}$, et al: Response inhibition rapidly increases singleneuron responses in the subthalamic nucleus of patients with Parkinson's disease. Cortex 84:111-123, 2016

4. Chastan N, Westby GWM, Yelnik J, Bardinet E, Do MC, Agid Y, et al: Effects of nigral stimulation on locomotion and postural stability in patients with Parkinson's disease. Brain 132:172-184, 2009

5. Georgopoulos AP, Kalaska JF, Caminiti R, Massey JT: On the relations between the direction of two-dimensional arm movements and cell discharge in primate motor cortex. J Neurosci 2:1527-1537, 1982

6. Georgopoulos AP, Schwartz AB, Kettner RE: Neuronal population coding of movement direction. Science 233:14161419,1986

7. Hanson TL, Fuller AM, Lebedev MA, Turner DA, Nicolelis MAL: Subcortical neuronal ensembles: an analysis of motor task association, tremor, oscillations, and synchrony in human patients. J Neurosci 32:8620-8632, 2012

8. Hausdorff JM, Gruendlinger L, Scollins L, O’Herron S, Tarsy D: Deep brain stimulation effects on gait variability in Parkinson's disease. Mov Disord 24:1688-1692, 2009

9. Hikosaka O, Isoda M: Switching from automatic to controlled behavior: cortico-basal ganglia mechanisms. Trends Cogn Sci 14:154-161, 2010

10. Hutchison WD, Allan RJ, Opitz H, Levy R, Dostrovsky JO, Lang AE, et al: Neurophysiological identification of the subthalamic nucleus in surgery for Parkinson's disease. Ann Neurol 44:622-628, 1998

11. Karimi M, Golchin N, Tabbal SD, Hershey T, Videen TO, $\mathrm{Wu}$ J, et al: Subthalamic nucleus stimulation-induced regional blood flow responses correlate with improvement of motor signs in Parkinson disease. Brain 131:2710-2719, 2008

12. Kishore S, Bagnall MW, McLean DL: Systematic shifts in the balance of excitation and inhibition coordinate the activity of axial motor pools at different speeds of locomotion. J Neurosci 34:14046-14054, 2014

13. Lohnes CA, Earhart GM: Effect of subthalamic deep brain stimulation on turning kinematics and related saccadic eye movements in Parkinson disease. Exp Neurol 236:389-394, 2012

14. Mack GA, Skillings JH: A Friedman-type rank test for main effects in a two-factor ANOVA. J Am Stat Assoc 75:947, 1980

15. Monchi O, Petrides M, Strafella AP, Worsley KJ, Doyon J: Functional role of the basal ganglia in the planning and execution of actions. Ann Neurol 59:257-264, 2006

16. Moran DW, Schwartz AB: Motor cortical representation of speed and direction during reaching. J Neurophysiol 82:2676-2692, 1999

17. Nambu A, Takada M, Inase M, Tokuno H: Dual somatotopical representations in the primate subthalamic nucleus: evidence for ordered but reversed body-map transformations from the primary motor cortex and the supplementary motor area. J Neurosci 16:2671-2683, 1996

18. Patil PG, Carmena JM, Nicolelis MAL, Turner DA: Ensemble recordings of human subcortical neurons as a source of motor control signals for a brain-machine interface. Neurosurgery 55:27-38, 2004

19. Piper M, Abrams GM, Marks WJ Jr: Deep brain stimulation for the treatment of Parkinson's disease: overview and impact on gait and mobility. NeuroRehabilitation 20:223-232, 2005

20. Rodriguez-Oroz MC, Rodriguez M, Guridi J, Mewes K, Chockkman V, Vitek J, et al: The subthalamic nucleus in Parkinson's disease: somatotopic organization and physiological characteristics. Brain 124:1777-1790, 2001

21. Rosin B, Slovik M, Mitelman R, Rivlin-Etzion M, Haber SN, Israel Z, et al: Closed-loop deep brain stimulation is superior in ameliorating parkinsonism. Neuron 72:370-384, 2011

22. Talpalar AE, Bouvier J, Borgius L, Fortin G, Pierani A, Kiehn O: Dual-mode operation of neuronal networks involved in left-right alternation. Nature 500:85-88, 2013

23. Tankus A, Fried I: Visuomotor coordination and motor representation by human temporal lobe neurons. J Cogn Neurosci 24:600-610, 2012

24. Tankus A, Fried I, Shoham S: Structured neuronal encoding and decoding of human speech features. Nat Commun 3:1015, 2012

25. Tankus A, Strauss I, Gurevich T, Mirelman A, Giladi N, Fried I, et al: Subthalamic neurons encode both single- and multi-limb movements in Parkinson's disease patients. Sci Rep 7:42467, 2017 
26. Tankus A, Yeshurun Y, Fried I: An automatic measure for classifying clusters of suspected spikes into single cells versus multiunits. J Neural Eng 6:056001, 2009

27. Theodosopoulos PV, Marks WJ Jr, Christine C, Starr PA: Locations of movement-related cells in the human subthalamic nucleus in Parkinson's disease. Mov Disord 18:791-798, 2003

28. Virtanen I: An entropy based correlation coefficient for categorized data, in Meristö T, Heinistö K (eds): Essays in Management Studies. Boston, 1983, pp 15-18 (http://lipas.uwasa. fi/ itv/publicat/boston.pdf) [Accessed February 15, 2018]

29. Wang C, Wai Y, Kuo B, Yeh YY, Wang J: Cortical control of gait in healthy humans: an fMRI study. J Neural Transm (Vienna) 115:1149-1158, 2008

30. Wichmann T, Bergman H, DeLong MR: The primate subthalamic nucleus. I. Functional properties in intact animals. J Neurophysiol 72:494-506, 1994

\section{Disclosures}

Dr. Giladi reports a consultant relationship with Teva-Lundbeck, Intec Pharma, NeuroDerm, Armon Neuromedical Ltd., Dexcel, Pharma Two B, UCB, Lysosomal Therapeutics Inc. (LTI), and Montfort; direct stock ownership in LTI; and membership on advisory boards of Teva-Lundbeck, LTI, Dexcel, AbbVie, NeuroDerm, and Sionara Mont4.

\section{Author Contributions}

Conception and design: all authors. Acquisition of data: Tankus, Fried. Analysis and interpretation of data: Tankus, Giladi, Hausdorff. Drafting the article: Tankus, Hausdorff. Critically revising the article: all authors. Reviewed submitted version of manuscript: Tankus. Statistical analysis: Tankus.

\section{Supplemental Information}

\section{Previous Presentations}

Portions of this work were presented in abstract form at the 20th International Congress of Parkinson's Disease and Movement Disorders, Berlin, Germany, June 2016; Karniel Computational Motor Control Workshop, Beersheba, Israel, June 2016; Congress of the International Society for Posture and Gait Research (ISPGR), Seville, Spain, June-July 2015; and Annual Meeting of Israel Society for Neuroscience, Eilat, Israel, December 2014.

\section{Correspondence}

Ariel Tankus: Tel Aviv Sourasky Medical Center, Tel Aviv, Israel. arielta@gmail.com. 